Supporting Information for

\title{
Temperature Dependence of Carbon-13 Kinetic Isotope Effects of Importance to Global Climate Change
}

Hai Lin, Yan Zhao, Benjamin A. Ellingson, Jingzhi Pu, and Donald G. Truhlar*

Department of Chemistry and Supercomputer Institute, University of Minnesota, Minneapolis, MN 55455-0431

Date of preparation of supporting information: Oct. 29, 2004

\section{Contents}

1. Table $\mathrm{S} 1$ gives rate constants for the reaction of ${ }^{12} \mathrm{CH}_{4}$ with $\mathrm{OH}$ radical at the MPW1K, MC3MPW, and MC3BB levels of theory.

2. Table S2 gives rate constants for the reaction of ${ }^{13} \mathrm{CH}_{4}$ with $\mathrm{OH}$ radical at the MPW1K, MC3MPW, and MC3BB levels of theory.

3. Table $\mathrm{S} 3$ gives the ${ }^{12} \mathrm{C} /{ }^{13} \mathrm{C}$ kinetic isotope effects for the reaction of $\mathrm{CH}_{4}$ with $\mathrm{OH}$ radical, as computed from Tables $\mathrm{S} 1$ and $\mathrm{S} 2$.

4. Table $\mathrm{S} 4$ gives the temperature dependence of the ${ }^{12} \mathrm{C} /{ }^{13} \mathrm{C}$ kinetic isotope effects of Tables S3 in parts per thousand (ppt). 
Table S1. Rate constants $\left(\mathrm{cm}^{3} /\right.$ molecule $\left.{ }^{-1} \mathrm{~s}^{-1}\right)$ for the reaction of ${ }^{12} \mathrm{CH}_{4}$ with $\mathrm{OH}$ radical ${ }^{a}$

\begin{tabular}{|c|c|c|c|c|}
\hline $\mathrm{T}(\mathrm{K})$ & TST & TST/W & CVT & $\mathrm{CVT} / \mathrm{SCT}$ \\
\hline \multicolumn{5}{|c|}{ MPW1K/MG3S } \\
\hline 175 & $1.354 \mathrm{E}-18$ & $9.610 \mathrm{E}-18$ & $6.322 \mathrm{E}-20$ & $1.048 \mathrm{E}-18$ \\
\hline 190 & $4.606 \mathrm{E}-18$ & $2.843 \mathrm{E}-17$ & $2.768 \mathrm{E}-19$ & $2.963 \mathrm{E}-18$ \\
\hline 200 & $9.407 \mathrm{E}-18$ & $5.331 \mathrm{E}-17$ & $6.549 \mathrm{E}-19$ & $5.542 \mathrm{E}-18$ \\
\hline 225 & $4.257 \mathrm{E}-17$ & $1.995 \mathrm{E}-16$ & $4.041 \mathrm{E}-18$ & $2.176 \mathrm{E}-17$ \\
\hline 250 & $1.431 \mathrm{E}-16$ & $5.706 \mathrm{E}-16$ & $1.739 \mathrm{E}-17$ & $6.788 \mathrm{E}-17$ \\
\hline 296 & $7.935 \mathrm{E}-16$ & $2.484 \mathrm{E}-15$ & $1.354 \mathrm{E}-16$ & $3.569 \mathrm{E}-16$ \\
\hline 300 & $8.995 \mathrm{E}-16$ & $2.765 \mathrm{E}-15$ & $1.573 \mathrm{E}-16$ & $4.039 \mathrm{E}-16$ \\
\hline 350 & $3.438 \mathrm{E}-15$ & $8.677 \mathrm{E}-15$ & $7.768 \mathrm{E}-16$ & $1.544 \mathrm{E}-15$ \\
\hline 400 & $9.660 \mathrm{E}-15$ & $2.093 \mathrm{E}-14$ & $2.611 \mathrm{E}-15$ & $4.372 \mathrm{E}-15$ \\
\hline 500 & $4.398 \mathrm{E}-14$ & $7.682 \mathrm{E}-14$ & $1.493 \mathrm{E}-14$ & $2.028 \mathrm{E}-14$ \\
\hline 600 & $1.302 \mathrm{E}-13$ & $1.977 \mathrm{E}-13$ & $5.024 \mathrm{E}-14$ & $6.073 \mathrm{E}-14$ \\
\hline 1000 & $1.656 \mathrm{E}-12$ & $1.965 \mathrm{E}-12$ & $7.532 \mathrm{E}-13$ & $7.786 \mathrm{E}-13$ \\
\hline \multicolumn{5}{|c|}{ MC3MPW } \\
\hline 175 & $3.243 \mathrm{E}-17$ & $1.790 \mathrm{E}-16$ & $1.432 \mathrm{E}-18$ & $1.300 \mathrm{E}-17$ \\
\hline 190 & $8.733 \mathrm{E}-17$ & $4.221 \mathrm{E}-16$ & $5.041 \mathrm{E}-18$ & $3.179 \mathrm{E}-17$ \\
\hline 200 & $1.557 \mathrm{E}-16$ & $6.943 \mathrm{E}-16$ & $1.051 \mathrm{E}-17$ & $5.457 \mathrm{E}-17$ \\
\hline 225 & $5.290 \mathrm{E}-16$ & $1.975 \mathrm{E}-15$ & $4.974 \mathrm{E}-17$ & $1.784 \mathrm{E}-16$ \\
\hline 250 & $1.415 \mathrm{E}-15$ & $4.547 \mathrm{E}-15$ & $1.733 \mathrm{E}-16$ & $4.802 \mathrm{E}-16$ \\
\hline 296 & $5.693 \mathrm{E}-15$ & $1.469 \mathrm{E}-14$ & $1.008 \mathrm{E}-15$ & $2.049 \mathrm{E}-15$ \\
\hline 300 & $6.305 \mathrm{E}-15$ & $1.600 \mathrm{E}-14$ & $1.146 \mathrm{E}-15$ & $2.283 \mathrm{E}-15$ \\
\hline 350 & $1.884 \mathrm{E}-14$ & $4.012 \mathrm{E}-14$ & $4.496 \mathrm{E}-15$ & $7.124 \mathrm{E}-15$ \\
\hline 400 & $4.396 \mathrm{E}-14$ & $8.199 \mathrm{E}-14$ & $1.274 \mathrm{E}-14$ & $1.790 \mathrm{E}-14$ \\
\hline 500 & $1.540 \mathrm{E}-13$ & $2.392 \mathrm{E}-13$ & $5.764 \mathrm{E}-14$ & $7.048 \mathrm{E}-14$ \\
\hline 600 & $3.817 \mathrm{E}-13$ & $5.285 \mathrm{E}-13$ & $1.667 \mathrm{E}-13$ & $1.894 \mathrm{E}-13$ \\
\hline 1000 & $3.378 \mathrm{E}-12$ & $3.846 \mathrm{E}-12$ & $1.873 \mathrm{E}-12$ & $1.904 \mathrm{E}-12$ \\
\hline \multicolumn{5}{|c|}{ MC3BB } \\
\hline 175 & $3.579 \mathrm{E}-17$ & $1.905 \mathrm{E}-16$ & $1.640 \mathrm{E}-18$ & $1.284 \mathrm{E}-17$ \\
\hline 190 & $9.529 \mathrm{E}-17$ & $4.448 \mathrm{E}-16$ & $5.687 \mathrm{E}-18$ & $3.188 \mathrm{E}-17$ \\
\hline 200 & $1.688 \mathrm{E}-16$ & $7.274 \mathrm{E}-16$ & $1.175 \mathrm{E}-17$ & $5.510 \mathrm{E}-17$ \\
\hline 225 & $5.653 \mathrm{E}-16$ & $2.044 \mathrm{E}-15$ & $5.451 \mathrm{E}-17$ & $1.818 \mathrm{E}-16$ \\
\hline 250 & $1.495 \mathrm{E}-15$ & $4.661 \mathrm{E}-15$ & $1.873 \mathrm{E}-16$ & $4.911 \mathrm{E}-16$ \\
\hline 296 & $5.919 \mathrm{E}-15$ & $1.486 \mathrm{E}-14$ & $1.064 \mathrm{E}-15$ & $2.084 \mathrm{E}-15$ \\
\hline 300 & $6.547 \mathrm{E}-15$ & $1.618 \mathrm{E}-14$ & $1.207 \mathrm{E}-15$ & $2.321 \mathrm{E}-15$ \\
\hline 350 & $1.932 \mathrm{E}-14$ & $4.021 \mathrm{E}-14$ & $4.663 \mathrm{E}-15$ & $7.431 \mathrm{E}-15$ \\
\hline 400 & $4.469 \mathrm{E}-14$ & $8.167 \mathrm{E}-14$ & $1.310 \mathrm{E}-14$ & $1.846 \mathrm{E}-14$ \\
\hline 500 & $1.546 \mathrm{E}-13$ & $2.365 \mathrm{E}-13$ & $5.828 \mathrm{E}-14$ & $7.104 \mathrm{E}-14$ \\
\hline 600 & $3.804 \mathrm{E}-13$ & $5.203 \mathrm{E}-13$ & $1.666 \mathrm{E}-13$ & $1.878 \mathrm{E}-13$ \\
\hline 1000 & $3.320 \mathrm{E}-12$ & $3.760 \mathrm{E}-12$ & $1.821 \mathrm{E}-12$ & $1.848 \mathrm{E}-12$ \\
\hline
\end{tabular}

${ }^{a}$ TST stands for conventional transition state theory, W for one-dimensional Wigner tunneling, CVT for canonical variational transition state theory, and SCT for multi-dimensional small curvature tunneling. All vibrational modes were treated harmonically in curvilinear coordinates except for the torsional mode that was treated by the hindered rotor RW scheme. 
Table S2. Rate constants $\left(\mathrm{cm}^{3} /\right.$ molecule $\left.\mathrm{s}^{1}\right)$ for the reaction of ${ }^{13} \mathrm{CH}_{4}$ with $\mathrm{OH}$ radical ${ }^{a}$

\begin{tabular}{|c|c|c|c|c|}
\hline $\mathrm{T}(\mathrm{K})$ & TST & TST/W & $\mathrm{CVT}$ & $\mathrm{CVT} / \mathrm{SCT}$ \\
\hline \multicolumn{5}{|c|}{ MPW1K/MG3S } \\
\hline 175 & $1.360 \mathrm{E}-18$ & $9.649 \mathrm{E}-18$ & $6.262 \mathrm{E}-20$ & $1.021 \mathrm{E}-18$ \\
\hline 190 & $4.625 \mathrm{E}-18$ & $2.854 \mathrm{E}-17$ & $2.744 \mathrm{E}-19$ & $2.900 \mathrm{E}-18$ \\
\hline 200 & $9.445 \mathrm{E}-18$ & $5.351 \mathrm{E}-17$ & $6.495 \mathrm{E}-19$ & $5.435 \mathrm{E}-18$ \\
\hline 225 & $4.274 \mathrm{E}-17$ & $2.003 \mathrm{E}-16$ & $4.011 \mathrm{E}-18$ & $2.142 \mathrm{E}-17$ \\
\hline 250 & $1.437 \mathrm{E}-16$ & $5.729 \mathrm{E}-16$ & $1.727 \mathrm{E}-17$ & $6.701 \mathrm{E}-17$ \\
\hline 296 & $7.973 \mathrm{E}-16$ & $2.496 \mathrm{E}-15$ & $1.347 \mathrm{E}-16$ & $3.533 \mathrm{E}-16$ \\
\hline 300 & $9.038 \mathrm{E}-16$ & $2.778 \mathrm{E}-15$ & $1.564 \mathrm{E}-16$ & $3.999 \mathrm{E}-16$ \\
\hline 350 & $3.457 \mathrm{E}-15$ & $8.723 \mathrm{E}-15$ & $7.733 \mathrm{E}-16$ & $1.531 \mathrm{E}-15$ \\
\hline 400 & $9.721 \mathrm{E}-15$ & $2.106 \mathrm{E}-14$ & $2.601 \mathrm{E}-15$ & $4.337 \mathrm{E}-15$ \\
\hline 500 & $4.432 \mathrm{E}-14$ & $7.741 \mathrm{E}-14$ & $1.487 \mathrm{E}-14$ & $2.013 \mathrm{E}-14$ \\
\hline 600 & $1.314 \mathrm{E}-13$ & $1.995 \mathrm{E}-13$ & $5.008 \mathrm{E}-14$ & $6.032 \mathrm{E}-14$ \\
\hline 1000 & $1.675 \mathrm{E}-12$ & $1.988 \mathrm{E}-12$ & $7.511 \mathrm{E}-13$ & $7.752 \mathrm{E}-13$ \\
\hline \multicolumn{5}{|c|}{ MC3MPW } \\
\hline 175 & $3.308 \mathrm{E}-17$ & $1.825 \mathrm{E}-16$ & $1.439 \mathrm{E}-18$ & $1.244 \mathrm{E}-17$ \\
\hline 190 & $8.900 \mathrm{E}-17$ & $4.301 \mathrm{E}-16$ & $5.068 \mathrm{E}-18$ & $3.070 \mathrm{E}-17$ \\
\hline 200 & $1.586 \mathrm{E}-16$ & $7.071 \mathrm{E}-16$ & $1.057 \mathrm{E}-17$ & $5.296 \mathrm{E}-17$ \\
\hline 225 & $5.383 \mathrm{E}-16$ & $2.009 \mathrm{E}-15$ & $5.006 \mathrm{E}-17$ & $1.747 \mathrm{E}-16$ \\
\hline 250 & $1.439 \mathrm{E}-15$ & $4.623 \mathrm{E}-15$ & $1.746 \mathrm{E}-16$ & $4.732 \mathrm{E}-16$ \\
\hline 296 & $5.786 \mathrm{E}-15$ & $1.492 \mathrm{E}-14$ & $1.015 \mathrm{E}-15$ & $2.034 \mathrm{E}-15$ \\
\hline 300 & $6.407 \mathrm{E}-15$ & $1.626 \mathrm{E}-14$ & $1.155 \mathrm{E}-15$ & $2.267 \mathrm{E}-15$ \\
\hline 350 & $1.914 \mathrm{E}-14$ & $4.076 \mathrm{E}-14$ & $4.531 \mathrm{E}-15$ & $7.095 \mathrm{E}-15$ \\
\hline 400 & $4.467 \mathrm{E}-14$ & $8.330 \mathrm{E}-14$ & $1.285 \mathrm{E}-14$ & $1.788 \mathrm{E}-14$ \\
\hline 500 & $1.565 \mathrm{E}-13$ & $2.431 \mathrm{E}-13$ & $5.813 \mathrm{E}-14$ & $7.066 \mathrm{E}-14$ \\
\hline 600 & $3.883 \mathrm{E}-13$ & $5.376 \mathrm{E}-13$ & $1.682 \mathrm{E}-13$ & $1.903 \mathrm{E}-13$ \\
\hline 1000 & $3.444 \mathrm{E}-12$ & $3.920 \mathrm{E}-12$ & $1.892 \mathrm{E}-12$ & $1.917 \mathrm{E}-12$ \\
\hline \multicolumn{5}{|c|}{ МС3BB } \\
\hline 175 & $3.642 \mathrm{E}-17$ & $1.939 \mathrm{E}-16$ & $1.645 \mathrm{E}-18$ & $1.258 \mathrm{E}-17$ \\
\hline 190 & $9.690 \mathrm{E}-17$ & $4.522 \mathrm{E}-16$ & $5.706 \mathrm{E}-18$ & $3.139 \mathrm{E}-17$ \\
\hline 200 & $1.715 \mathrm{E}-16$ & $7.392 \mathrm{E}-16$ & $1.179 \mathrm{E}-17$ & $5.438 \mathrm{E}-17$ \\
\hline 225 & $5.740 \mathrm{E}-16$ & $2.075 \mathrm{E}-15$ & $5.476 \mathrm{E}-17$ & $1.803 \mathrm{E}-16$ \\
\hline 250 & $1.517 \mathrm{E}-15$ & $4.730 \mathrm{E}-15$ & $1.882 \mathrm{E}-16$ & $4.885 \mathrm{E}-16$ \\
\hline 296 & $6.004 \mathrm{E}-15$ & $1.508 \mathrm{E}-14$ & $1.070 \mathrm{E}-15$ & $2.080 \mathrm{E}-15$ \\
\hline 300 & $6.641 \mathrm{E}-15$ & $1.641 \mathrm{E}-14$ & $1.214 \mathrm{E}-15$ & $2.317 \mathrm{E}-15$ \\
\hline 350 & $1.960 \mathrm{E}-14$ & $4.078 \mathrm{E}-14$ & $4.693 \mathrm{E}-15$ & $7.434 \mathrm{E}-15$ \\
\hline 400 & $4.533 \mathrm{E}-14$ & $8.284 \mathrm{E}-14$ & $1.319 \mathrm{E}-14$ & $1.849 \mathrm{E}-14$ \\
\hline 500 & $1.569 \mathrm{E}-13$ & $2.400 \mathrm{E}-13$ & $5.871 \mathrm{E}-14$ & $7.128 \mathrm{E}-14$ \\
\hline 600 & $3.864 \mathrm{E}-13$ & $5.285 \mathrm{E}-13$ & $1.679 \mathrm{E}-13$ & $1.887 \mathrm{E}-13$ \\
\hline 1000 & $3.380 \mathrm{E}-12$ & $3.827 \mathrm{E}-12$ & $1.838 \mathrm{E}-12$ & $1.860 \mathrm{E}-12$ \\
\hline
\end{tabular}

${ }^{a}$ TST stands for conventional transition state theory, W for one-dimensional Wigner tunneling, CVT for canonical variational transition state theory, and SCT for multi-dimensional small curvature tunneling. All vibrational modes were treated harmonically in curvilinear coordinates except for the torsional mode that was treated by the hindered rotor RW scheme. 
Table S3. The ${ }^{12} \mathrm{C} /{ }^{13} \mathrm{C}$ kinetic isotope effects for the reaction of $\mathrm{CH}_{4}$ with $\mathrm{OH}$ radical ${ }^{a}$

\begin{tabular}{|c|c|c|c|c|}
\hline $\mathrm{T}(\mathrm{K})$ & TST & TST/W & CVT & $\mathrm{CVT} / \mathrm{SCT}$ \\
\hline \multicolumn{5}{|c|}{ MPW1K/MG3S } \\
\hline 175 & 0.9957 & 0.9960 & 1.0095 & 1.0266 \\
\hline 190 & 0.9959 & 0.9961 & 1.0087 & 1.0220 \\
\hline 200 & 0.9959 & 0.9962 & 1.0083 & 1.0197 \\
\hline 225 & 0.9959 & 0.9962 & 1.0074 & 1.0156 \\
\hline 250 & 0.9958 & 0.9960 & 1.0066 & 1.0130 \\
\hline 296 & 0.9953 & 0.9955 & 1.0056 & 1.0101 \\
\hline 300 & 0.9952 & 0.9954 & 1.0056 & 1.0100 \\
\hline 350 & 0.9945 & 0.9947 & 1.0046 & 1.0085 \\
\hline 400 & 0.9937 & 0.9939 & 1.0041 & 1.0079 \\
\hline 500 & 0.9923 & 0.9924 & 1.0036 & 1.0075 \\
\hline 600 & 0.9911 & 0.9912 & 1.0033 & 1.0069 \\
\hline 1000 & 0.9883 & 0.9883 & 1.0028 & 1.0043 \\
\hline \multicolumn{5}{|c|}{ MC3MPW } \\
\hline 175 & 0.9803 & 0.9804 & 0.9952 & 1.0448 \\
\hline 190 & 0.9813 & 0.9814 & 0.9946 & 1.0353 \\
\hline 200 & 0.9817 & 0.9819 & 0.9943 & 1.0303 \\
\hline 225 & 0.9827 & 0.9829 & 0.9936 & 1.0210 \\
\hline 250 & 0.9834 & 0.9835 & 0.9931 & 1.0147 \\
\hline 296 & 0.9841 & 0.9842 & 0.9923 & 1.0075 \\
\hline 300 & 0.9841 & 0.9843 & 0.9923 & 1.0071 \\
\hline 350 & 0.9843 & 0.9844 & 0.9923 & 1.0041 \\
\hline 400 & 0.9842 & 0.9843 & 0.9920 & 1.0011 \\
\hline 500 & 0.9836 & 0.9837 & 0.9914 & 0.9974 \\
\hline 600 & 0.9830 & 0.9831 & 0.9909 & 0.9952 \\
\hline 1000 & 0.9811 & 0.9811 & 0.9895 & 0.9930 \\
\hline \multicolumn{5}{|c|}{ MC3BB } \\
\hline 175 & 0.9826 & 0.9827 & 0.9971 & 1.0208 \\
\hline 190 & 0.9834 & 0.9836 & 0.9965 & 1.0158 \\
\hline 200 & 0.9839 & 0.9840 & 0.9962 & 1.0132 \\
\hline 225 & 0.9848 & 0.9849 & 0.9955 & 1.0085 \\
\hline 250 & 0.9853 & 0.9855 & 0.9950 & 1.0053 \\
\hline 296 & 0.9859 & 0.9860 & 0.9941 & 1.0018 \\
\hline 300 & 0.9859 & 0.9860 & 0.9942 & 1.0016 \\
\hline 350 & 0.9860 & 0.9860 & 0.9936 & 0.9997 \\
\hline 400 & 0.9858 & 0.9859 & 0.9933 & 0.9984 \\
\hline 500 & 0.9852 & 0.9852 & 0.9927 & 0.9966 \\
\hline 600 & 0.9844 & 0.9845 & 0.9923 & 0.9955 \\
\hline 1000 & 0.9824 & 0.9824 & 0.9910 & 0.9931 \\
\hline
\end{tabular}

${ }^{a}$ Computed from Tables S1 and S2. TST stands for conventional transition state theory, W for one-dimensional Wigner tunneling, CVT for canonical variational transition state theory, and SCT for multi-dimensional small curvature tunneling. 
Table S4. The temperature dependence of the KIEs of Table S3 in parts per thousand ${ }^{a}$

\begin{tabular}{|c|c|c|c|c|}
\hline $\mathrm{T}(\mathrm{K})$ & TST & $\mathrm{TST} / \mathrm{W}$ & CVT & $\mathrm{CVT} / \mathrm{SCT}$ \\
\hline \multicolumn{5}{|c|}{ MPW1K/MG3S } \\
\hline 175 & -0.4 & -0.5 & -4.0 & -16 \\
\hline 190 & -0.6 & -0.7 & -3.2 & -12 \\
\hline 200 & -0.7 & -0.7 & -2.7 & -9.5 \\
\hline 225 & -0.7 & -0.7 & -1.8 & -5.5 \\
\hline 250 & -0.5 & -0.6 & -1.0 & -2.9 \\
\hline 296 & 0.0 & 0.0 & 0.0 & 0.0 \\
\hline 300 & -0.1 & 0.0 & 0.0 & -0.2 \\
\hline 350 & -0.8 & -0.8 & -1.0 & -1.6 \\
\hline 400 & -1.6 & -1.6 & -1.4 & -2.2 \\
\hline 500 & -3.0 & -3.1 & -1.9 & -2.6 \\
\hline 600 & -4.2 & -4.3 & -2.3 & -3.2 \\
\hline 1000 & -7.0 & -7.2 & -2.8 & -5.8 \\
\hline \multicolumn{5}{|c|}{ MC3MPW } \\
\hline 175 & 3.8 & 3.8 & -2.9 & -37 \\
\hline 190 & 2.9 & 2.8 & -2.3 & -28 \\
\hline 200 & 2.4 & 2.3 & -2.0 & -23 \\
\hline 225 & 1.3 & 1.3 & -1.3 & -13 \\
\hline 250 & 0.7 & 0.7 & -0.8 & -7.1 \\
\hline 296 & 0.0 & 0.0 & 0.0 & 0.0 \\
\hline 300 & 0.0 & 0.1 & 0.0 & -0.4 \\
\hline 350 & 0.2 & 0.2 & 0.0 & -3.4 \\
\hline 400 & 0.1 & 0.1 & -0.3 & -6.4 \\
\hline 500 & -0.4 & -0.5 & -0.9 & -10 \\
\hline 600 & -1.1 & -1.1 & -1.4 & -12 \\
\hline 1000 & -3.0 & -3.2 & -2.8 & -15 \\
\hline \multicolumn{5}{|c|}{ MC3BB } \\
\hline 175 & 3.3 & 3.3 & -3.0 & -19 \\
\hline 190 & 2.5 & 2.5 & -2.5 & -14 \\
\hline 200 & 2.0 & 2.0 & -2.1 & -11 \\
\hline 225 & 1.1 & 1.1 & -1.4 & -6.7 \\
\hline 250 & 0.6 & 0.6 & -0.8 & -3.5 \\
\hline 296 & 0.0 & 0.0 & 0.0 & 0.0 \\
\hline 300 & 0.0 & 0.0 & 0.0 & -0.2 \\
\hline 350 & 0.1 & 0.0 & -0.5 & -2.2 \\
\hline 400 & -0.1 & -0.1 & -0.9 & -3.4 \\
\hline 500 & -0.7 & -0.8 & -1.4 & -5.2 \\
\hline 600 & -1.5 & -1.5 & -1.9 & -6.3 \\
\hline 1000 & -3.5 & -3.6 & -3.1 & -8.7 \\
\hline
\end{tabular}

${ }^{a}$ Computed from Table S3 as $\left\{\left[\operatorname{KIE}\left(T_{0}\right)-\operatorname{KIE}(T)\right] / \operatorname{KIE}\left(T_{0}\right)\right\} \times 1000$ if $T<T_{0}$ and as $\{[\operatorname{KIE}(T)-$ $\left.\left.\operatorname{KIE}\left(T_{0}\right)\right] / \operatorname{KIE}\left(T_{0}\right)\right\} \times 1000$ if $T \geq T_{0}$, where $T_{0}=296 \mathrm{~K}$. TST stands for conventional transition state theory, $\mathrm{W}$ for one-dimensional Wigner tunneling, CVT for canonical variational transition state theory, and SCT for multi-dimensional small curvature tunneling. 